\title{
Silk-Reinforced Collagen Hydrogels with Raised Multiscale Stiffness for Mesenchymal Cells 3D Culture
}

\author{
Hector Sanz-Fraile, MSc, ${ }^{1}$ Susana Amoros, MSc, ${ }^{2}$ Irene Mendizabal, MSc, ${ }^{1}$ Carolina Galvez-Monton, PhD, ${ }^{2-4}$ \\ Cristina Prat-Vidal, PhD, ${ }^{2-5}$ Antoni Bayes-Genis, MD, PhD, ${ }^{2-4,6}$ Daniel Navajas, PhD, ${ }^{1,7,8}$ \\ Ramon Farre, $\mathrm{PhD},{ }^{1,7,9}$ and Jorge Otero, $\mathrm{PhD}^{1,7}$
}

Type I collagen hydrogels are of high interest in tissue engineering. With the evolution of 3D bioprinting technologies, a high number of collagen-based scaffolds have been reported for the development of 3D cell cultures. A recent proposal was to mix collagen with silk fibroin derived from Bombyx mori silkworm. Nevertheless, due to the difficulties in the preparation and the characteristics of the protein, several problems such as phase separation and collagen denaturation appear during the procedure. Therefore, the common solution is to diminish the concentration of collagen although in that way the most biologically relevant component is reduced. In this study, we present a new, simple, and effective method to develop a collagen-silk hybrid hydrogel with high collagen concentration and with increased stiffness approaching that of natural tissues, which could be of high interest for the development of cardiac patches for myocardial regeneration and for preconditioning of mesenchymal stem cells (MSCs) to improve their therapeutic potential. Sericin in the silk was preserved by using a physical solubilizing procedure that results in a preserved fibrous structure of type I collagen, as shown by ultrastructural imaging. The macro- and micromechanical properties of the hybrid hydrogels measured by tensile stretch and atomic force microscopy, respectively, showed a more than twofold stiffening than the collagen-only hydrogels. Rheological measurements showed improved printability properties for the developed biomaterial. The suitability of the hydrogels for 3D cell culture was assessed by 3D bioprinting bone marrow-derived MSCs cultured within the scaffolds. The result was a biomaterial with improved printability characteristics that better resembled the mechanical properties of natural soft tissues while preserving biocompatibility owing to the high concentration of collagen.

Keywords: collagen, silk, hydrogel, mesenchymal cells, 3D bioprinting, multiscale mechanics

\section{Impact Statement}

In this study, we report the development of silk microfiber-reinforced type I collagen hydrogels for 3D bioprinting and cell culture. In contrast with previously reported studies, a novel physical method allowed the preservation of the silk sericin protein. Hydrogels were stable, showed no phase separation between the biomaterials, and they presented improved printability. An increase between two- and threefold of the multiscale stiffness of the scaffolds was achieved with no need of using additional crosslinkers or complex methods, which could be of high relevance for cardiac patches development and for preconditioning mesenchymal stem cells (MSCs) for therapeutic applications. We demonstrate that bone marrowderived MSCs can be effectively bioprinted and 3D cultured within the stiffened structures.

\footnotetext{
${ }^{1}$ Unit of Biophysics and Bioengineering, School of Medicine and Health Sciences, Universitat de Barcelona, Barcelona, Spain.

${ }^{2}$ ICREC Research Program, Germans Trias i Pujol Health Science Research Institute, Badalona, Spain.

${ }^{3}$ Hearth Institute (iCor), Germans Trias i Pujol University Hospital, Badalona, Spain.

${ }_{5}^{4}$ CIBER Cardiovascular, Instituto de Salud Carlos III, Madrid, Spain.

${ }^{5}$ Institut d'Investigació Biomèdica de Bellvitge, IDIBELL, L'Hospitalet de Llobregat, Spain.

${ }^{6}$ Department of Medicine, Autonomous University of Barcelona, Barcelona, Spain.

${ }^{7}$ CIBER de Enfermedades Respiratorias, Instituto de Salud Carlos III, Madrid, Spain.

${ }^{8}$ Institute for Bioengineering of Catalonia (IBEC), The Barcelona Institute of Science and Technology, Barcelona, Spain.

${ }^{9}$ Institut d'investigacions Biomèdiques Agustí Pi i Sunyer, Barcelona, Spain.
} 


\section{Introduction}

$\mathbf{H}$ YDROGELS ARE HYDROPHILIC polymer networks holding vast quantities of water and presenting mechanical properties similar to those found in soft biological tissues. With the development of 3D bioprinting technology, which allows for customized and high-throughput development of scaffolds, the research interest in naturally derived hydrogels for tissue engineering and regenerative medicine applications has increased dramatically. Scaffolds based on cell-laden hydrogels can be 3D printed layer-by-layer using different technical approaches. ${ }^{1}$ Hydrogels mimicking native soft tissue microenvironments and 3D constructs with different shapes can be fabricated in an automated way. In recent years, a high number of different biomaterials have been used as 3D scaffolds for cell culturing and for 3D bioprinting. ${ }^{2}$

Naturally derived type I collagen hydrogels are widely employed since they are biocompatible and biodegradable and, importantly, because they can modulate cell function because their fibrous structure of binding ligands to cell surface receptors. ${ }^{3}$ Collagen I hydrogels have the drawback that they experience considerable volume reduction when cells are cultured within them. ${ }^{4}$ Another limitation of collagen I hydrogels is that they exhibit a stiffness (a few hundreds of $\mathrm{Pa}$ ) that is lower than natural tissues such as those in the lung and heart. Given that mesenchymal stem cells (MSCs) are mechanosensitive, ${ }^{5}$ it is important to culture them in a physiomimetic mechanical environment, which requires a 3D substrate stiffer than conventional collagen I hydrogels.

Modification of the mechanical properties of the 3D scaffolds where MSCs are cultured is a growing research area for several tissue engineering applications. In vivo, one of the most important applications is the generation of cardiac patches for myocardial infarction therapy. ${ }^{6}$ Although MSCs have very limited capacity for cardiac differentiation, they have a therapeutic impact in cardiac regeneration mainly due to their immunosuppressive profile and paracrine effects. ${ }^{7}$ Scaffolds are needed because it has been shown that only a small percentage of the transplanted cells (by injection or perfusion) engrafts into the host myocardium, with the result that the duration of the therapeutic secreted factors is quite limited ${ }^{8}$ if the cells are transplanted without a scaffold for retaining them close to the damaged tissue. Commercially available cardiac patches are based in glutaraldehyde-fixed bovine pericardium, ${ }^{9}$ but they present several limitations and $\sim 25 \%$ of patients need a second surgery after a short period of time. ${ }^{10}$ Accordingly, considerable research is currently developed in this field.

In vitro, it is well known that the secretion of several cytokines is regulated by the stiffness of the microenvironment where MSCs are cultured. ${ }^{11}$ In cardiac tissue engineering, it has been shown that differentiation requires stiffer scaffolds (with elastic modulus in the range of hundreds of $\mathrm{kPa}$ ) while beating behavior is better accomplished with softer scaffolds (elastic modulus in the range of $\mathrm{kPa}$ ). ${ }^{12}$ Therefore, the tunability of the mechanical behavior of scaffold is of high importance for the in vitro culture of MSCs aimed at therapeutic applications, ${ }^{13}$ for instance when culturing cells for cardiac patches where their paracrine effects should be enhanced while differentiation is not an objective.
3D bioprinting is considerably modifying the production processes of scaffolds as it offers the possibility to fabricate a high number of replicates, thus reducing the batch-tobatch variability. ${ }^{14}$ Thus, besides having the desired mechanical properties, hydrogels for cardiac patches and for scaffolds to precondition MSCs should be easily bioprintable. Stiffer collagen hydrogels can be obtained by increasing protein concentration, ${ }^{15}$ although this procedure makes difficult their manipulation and 3D printability because of a dramatic decrease in gelation time. ${ }^{16}$ Moreover, their viscosity in the pregel phase is not stable, which makes difficult to set up the bioprinter as the pressure applied needs to be adapted during the printing process.

Another possible strategy to obtain stiffer hydrogels is by means of physical (usually with UV radiation) or chemical crosslinking the collagen structure. ${ }^{17}$ Nevertheless, such crosslinking processes are known to modify the physiological cell response (or even to be cytotoxic) when culturing MSCs within the scaffolds. ${ }^{18}$ However, a common strategy to increase hydrogel stiffness is to mix collagen with other natural or synthetic biomaterials, being alginate, ${ }^{19}$ polyethylene glycol, and polycaprolactone ${ }^{20}$ the most widely employed.

Recently, silk-derived materials have started to be used with this purpose. ${ }^{21-23}$ Although it is possible to obtain silk from spiders, ${ }^{24}$ the vast majority of reported studies use silk derived from the Bombyx mori silkworm. This material has been used for years in the textile industry, so it is cheap and highly available, and it also has shown its potential as biomaterial for tissue engineering ${ }^{25,26}$ due to its high biocompatibility, which is comparable with that of polylactic acid and collagen. ${ }^{27-29}$ Silk is a fibrous protein mainly composed of fibroin and sericin. The majority of studies in tissue engineering have used fibroin, ${ }^{30}$ because sericin was supposed to have immunogenic activity. ${ }^{31}$ Nevertheless, more recent studies have shown no clear differences in immunological response when using silk fibroin or sericin. ${ }^{32}$ Moreover, some studies are presenting sericin as a highly interesting biomaterial, mainly due to its positive effects in mesenchymal cells like fibroblasts. 33,34

The aim of this study was to improve the integration between collagen and sericin-preserved silk to obtain a useful bioink for 3D bioprinting hydrogel scaffolds to be potentially applied in tissue engineering and regenerative medicine, such as the development of stiffened cardiac patches and new microenvironments for the preconditioning of MSCs. To this end, we designed and tested a new, simple, and effective method to dissolve raw silkworm cocoons by physically milling them into liquid nitrogen $\left(\mathrm{LN}_{2}\right)$ and using $\mathrm{NaOH}$ as solvent. The ultrastructure of the resulting hydrogels and their macro- and microscale properties were assessed by scanning electron microscopy (SEM), tensile stretch (TS) tests, and atomic force microscopy (AFM), respectively. The culture of 3D bioprinted bone marrow-derived MSCs (BM-MSCs) within the resulting scaffolds was also tested.

\section{Materials and Methods}

\section{Preparation of silk microfiber-reinforced type I collagen hydrogels}

All animal care and experimental procedures were approved by the ethical committee for animal research of the University of Barcelona. Rat-tail type I collagen was 
extracted by following the protocol described in Rajan et al. $^{35}$ In brief, tails from Sprague-Dawley rats (male, $350 \mathrm{~g}$ ) were collected as a by-product from other experiments in the animal facilities of the School of Medicine. Tendons were extracted from the tails, rinsed with phosphate-buffered saline (PBS), acetone, and isopropanol, and then dissolved in $0.02 \mathrm{~N}$ acetic acid at $4^{\circ} \mathrm{C}$ for $48 \mathrm{~h}$. The solution was then freeze dried (Telstar Lyoquest-55 Plus, Terrassa, Spain), and the resulting sponge was solubilized in $0.02 \mathrm{~N}$ acetic acid at a concentration of $10 \mathrm{mg} / \mathrm{mL}$ and stored at $4{ }^{\circ} \mathrm{C}$ for further use.

Silk was obtained from raw (sericin-preserved) B. mori silkworm cocoons (AYARA Health, Yangon, Myanmar). Cocoons were rinsed in deionized (DI) water and then milled in $\mathrm{LN}_{2}$ by using a cryogenic mill (SPEX 6755, NJ) to obtain a micron-sized powder and stored at $-80^{\circ} \mathrm{C}$ for further use. To prepare the pregels, powder was dissolved in $1 \mathrm{M} \mathrm{NaOH}$ under magnetic stirring until no particles were visible.

Pregel mixtures were prepared by stabilizing the $\mathrm{pH}$ of the type I collagen to $7.4 \pm 0.4$ with $1 \mathrm{M} \mathrm{NaOH}$, and by stabilizing the $\mathrm{pH}$ of the silk solution with $2 \mathrm{M} \mathrm{HCl}$ for a final silk concentration of $70 \mathrm{mg} / \mathrm{mL}$. For gelation, the collagen pregel was mixed with ice-cold $\mathrm{pH}$-neutralized silk at different ratios of collagen-silk protein $(4: 1,2: 1,4: 3$, and $1: 1$, which will be referred as $25 \%, 50 \%, 75 \%$, and $100 \%$ of silk content with respect to the collagen, respectively), resulting in a final concentration of the collagen protein of $7.8 \mathrm{mg} / \mathrm{mL}$. For 3D structures formation, the pregels were incubated at $37^{\circ} \mathrm{C}$ for $45 \mathrm{~min}$ to jellify.

\section{Bioink development}

Human BM-MSCs (ATCC, VA) were expanded following the manufacturer's instructions. Expanded BM-MSCs were trypsinized and resuspended in culture media $\left(1 \times 10^{6}\right.$ cells $/ \mathrm{mL}$ ) and mixed with the collagen control, $50 \%$, and $100 \%$ silk-containing collagen pregels before 3D bioprinting ( $n=3$ for each concentration). Control BM-MSCs were cultured on standard plastic substrate. All experiments were conducted with cells at passages $2-4$.

\section{Ultrastructure characterization by scanning electron microscopy imaging}

The ultrastructure of the constructs was characterized using SEM by adapting the protocol described in Wolf et al. $^{36}$ In brief, acellular and BM-MSCs laden hydrogel scaffolds (cells cultured for $48 \mathrm{~h}$ ) containing $0 \%, 50 \%$, and $100 \%$ weight silk content with respect to the collagen protein were fixed for $48 \mathrm{~h}$ in $4 \%$ paraformaldehyde in PBS and then washed three times in $0.1 \mathrm{M}$ phosphate buffer for $10 \mathrm{~min}$ each and then incubated in $4 \% \mathrm{OsO}_{4}$ for $90 \mathrm{~min}$. The next step was a series of washes with DI water until there was no $\mathrm{OsO}_{4}$ inside the container where the samples were hanged on. Then, samples were dehydrated in ethanol solutions, with increasing concentrations from $50 \%$ till absolute ethanol at $4{ }^{\circ} \mathrm{C}$, and critical point dried (autosamdri-815 critical point dryer; Tousimis, Rockville, MD). Samples were mounted using conductive adhesive tabs (TED PELLA) and they were carbon coated before imaging with a JSM-6510 (JEOL, Tokyo, Japan) SEM at $3 \mathrm{kV}$.

\section{Swelling and degradation}

Swelling and degradation of the scaffolds were studied as described in Wang et al. ${ }^{37}$ After preparation of the three replicates for each condition of the hydrogels, they were weighted, freeze dried, and immersed in PBS at $\mathrm{pH}$ 7.4. During 3 weeks, hydrogels were taken out at certain time points and weighted to determine the amount of water, and the results are expressed as the percentage with respect to the original weight before freeze drying.

\section{Measurement of microscale mechanical properties by atomic force microscopy}

The microscale stiffness of silk-containing acellular hydrogels was measured by AFM. Hydrogels with specific geometries for these measurements were developed by bioprinting molds in F-127 hydrogel (pluronic) (3D Discovery bioprinter; RegenHU, Switzerland). Squared structures $(5 \times 5 \times$ $0.1 \mathrm{~mm}$ ) were bioprinted on top of positively charged glass slides and then the pregels were casted into them. After gelation, pluronic was removed by immersion in PBS at $4^{\circ} \mathrm{C}$ for $10 \mathrm{~min}$. All measurements were performed inside a bath with PBS at $37^{\circ} \mathrm{C}$.

Three batches of three replicates for each sample were fabricated for each silk-collagen mixture of the hydrogels. Measurements were conducted by using a custom-built AFM mounted on an inverted optical microscope (TE2000; Nikon, Tokyo, Japan) equipped with a $\mathrm{V}$-shaped silicon nitride cantilevers $(0.03 \mathrm{~N} / \mathrm{m}$ nominal spring constant) ended with a $5 \mu \mathrm{m}$ radius spherical polystyrene bead (Novascan Technologies, Ames, IA). Elastic modulus was computed from the force-displacement curves by adjusting the Hertz model as described in Alcaraz et al. ${ }^{38}$ The micromechanics of each sample were measured in four randomly selected zones. Five force curves ( $1 \mathrm{~Hz}$ and $10 \mu \mathrm{m}$ amplitude) in four points randomly selected and separated $\sim 50-100 \mu \mathrm{m}$ from each other were recorded in each zone.

Micromechanical stiffness $\left(E_{m}\right)$ of each sample was characterized as the average from the different curves recorded in the sample. The values for each batch were calculated as the average of the measurement of three samples $(n=3)$.

\section{Assessment of macroscale mechanical properties by tensile stretch and bulk compression tests}

For TS, three batches of four strips of hydrogels, each $5 \times 2 \times 2 \mathrm{~mm}$, were fabricated for each concentration mixture condition of the collagen-silk hydrogels following the same procedure than for the AFM samples. One end of the strip was glued with cyanoacrylate to a small hook attached to the lever of a servo-controlled displacement actuator with an integrated force sensor (300C-LR; Aurora Scientific, Aurora, Canada), which permitted stretching the strip and measuring both the stretched length and the applied force simultaneously. The other end of the strip was glued to a fixed hook. The stress and the elastic modulus at $20 \%$ of stretch of each strip were computed from a series of 10 forcedisplacement curves at $0.2 \mathrm{~Hz}$ frequency and $30 \%$ strain, as described in Farré et al. ${ }^{39}$

In brief, the strain-stress curves were calculated from the force-displacement curves using geometrical measurements to calculate the cross section, and the macroscale elastic modulus $\left(E_{M}\right)$ was computed by local derivation of the curve around the $20 \%$ stretching point. The values for each batch were calculated as the average of the measurement of four strip replicates $(n=3)$. 
For bulk compression measurements, a batch of three collagen-silk hydrogels was fabricated for the control, $50 \%$, and $100 \%$ mixtures. Each hydrogel was shaped as a cylinder of $15.6 \mathrm{~mm}$ base and $10 \mathrm{~mm}$ height and covered with a $16 \mathrm{~mm}$ flat-ended cylindrical indentator. Compression modulus was measured by applying a compressive force with a custom-made base indentator system attached to a servocontrolled displacement actuator with an integrated force sensor (305C; Aurora Scientific), which permitted compressing the sample and measuring both the compressed length and the applied force simultaneously.

A series of 10 force-displacement curves at $0.2 \mathrm{~Hz}$ frequency were measured using this system for each sample: the first and last curves were discarded for cohesion purposes. The elastic modulus at compression of each sample was computed from the mean of those eight curves given the linear relationship between the penetration and the mean contact pressure due to the constant contact area by using Eq. (1).

$$
P_{m}=\frac{2 E h}{\pi a\left(1-v^{2}\right)},
$$

where $P_{m}$ is the mean contact pressure, $E$ the elastic modulus of the sample, $h$ the penetration, $a$ the radius of the indenter, and $v$ the Poisson ratio (assumed 0.5).

\section{Printability characterization}

Hydrogel rheological properties were measured by using a HAAKE RheoStress 1 rheometer (ThermoFisher, MA) with $35 \mathrm{~mm}$ serrated parallel plate geometry. Hydrogels were neutralized just before mechanical testing was done. To test the hydrogels, pregel solution was loaded onto a Peltier plate set at $4^{\circ} \mathrm{C}$ and with a distance between plates of $200 \mu \mathrm{m}$. The storage modulus $\left(G^{\prime}\right)$, loss modulus $\left(G^{\prime \prime}\right)$, and dynamic viscosity $(\mu)$ were measured at a constant $0.1 \mathrm{~Hz}$ with a strain of $5 \%$. The temperature of the plates was constant at $4{ }^{\circ} \mathrm{C}$ for $15 \mathrm{~min}$, then increased to $37^{\circ} \mathrm{C}$, and held constant for $15 \mathrm{~min}$ more.

For 3D bioprinting, a cartridge of the 3D bioprinter (3Ddiscovery; RegenHU) was filled with the pregel solution mixed with the cells and maintained at $4^{\circ} \mathrm{C}$ during all the printing process. A secondary printing cartridge was filled with Pluronic F127 gel (40\% v/v in PBS) at room temperature. Hydrogels were printed at $\sim 2$ bar of pressure using a nozzle of $330 \mu \mathrm{m}$ (Nordson EFD) and F127 was printed at $\sim 4.5$ bar using a needle of $200 \mu \mathrm{m}$ (Nordson EFD). The 3D structures were then constructed layer-by-layer by alternatively printing an F127 layer, which served as a template, and a pregel layer that filled the F127 template layer. After the last layer was printed, the 3D structures were incubated at $37^{\circ} \mathrm{C}$ to form the hydrogel. F127 structure was finally dissolved by immersing the structures in culture media at $4^{\circ} \mathrm{C}$ for $10 \mathrm{~min}$.

\section{D bioprinted cell cultures}

BM-MSCs were cultured within the scaffolds and imaged after staining them with a Live/Dead viability kit (ThermoFischer) following the manufacturer's instructions. Cells were stained with calcein AM (live cells, green) and EthD-1 (dead cells, red) after $4 \mathrm{~h}$ and 7 days of $3 \mathrm{D}$ bioprinting the scaffolds (1.5 $\mathrm{mm}$ in height). As silk presented high autofluorescence in the red channel, only qualitative analysis of the green channel of the images was done. For morphological analysis of the cells within the hydrogels, structures at $48 \mathrm{~h}$ were fixed in $4 \%$ paraformaldehyde for $48 \mathrm{~h}$ and then immersed in optimal cutting temperature compound (OCT; Sigma) and frozen at $-80^{\circ} \mathrm{C}$. Thin tissue slices $(\sim 70 \mu \mathrm{m})$ were obtained by cryosectioning (CM3050; Leica Microsystems, Germany) and placed on top of positively charged glass slides. OCT was removed by thawing and washing the samples in PBS solution at room temperature. Samples were then stained for DNA (NucBlue) and F-actin (phalloidin). Stained scaffolds were then imaged by confocal microscopy (TI-HUBC; Nikon) with a $60 \times$ objective.

\section{Statistical analysis}

Data are expressed as mean \pm standard error. One-way analysis of variance (ANOVA) tests were performed to compare changes induced by the different concentrations of silk in the mixture. Statistical significance was considered at $p$-values $<0.05$.

\section{Results}

\section{Ultrastructure of the scaffolds}

Macroscopic images showed no phase separation between both materials in the hydrogels (Fig. 1a). The ultrastructural images (Fig. 1b, c) showed a good integration of BM-MSCs within the scaffolds, as well as the incorporation of the silk fibers in the collagen matrix of the hydrogels. Formation of collagen microfibrils $(\sim 100-200 \mathrm{~nm})$ did not seem to be affected by the incorporation of the silk fibers $(\sim 5-10 \mu \mathrm{m})$.

\section{Swelling and degradation}

Hydrogels formed purely from collagen or with a 50\% silk content recovered $70-80 \%$ of their original weight, whereas hydrogels with $100 \%$ of silk content recovered their full weight after a couple of days. There was no observable degradation of the hydrogels after that during the 3 weeks of the experiment.

\section{Microscale mechanical properties}

The microscale elastic moduli of the hydrogels were increased by about twofold in the silk-collagen mixture as compared with pure collagen, increasing with silk concentration up to a maximum that was found at the $50 \%$ and $100 \%$ of silk content $\left(E_{m}=1.26 \pm 0.17 \mathrm{kPa}\right.$ and $1.31 \pm 0.23 \mathrm{kPa}$, respectively) with respect to the pure collagen control $\left(E_{m}=\right.$ $0.62 \pm 0.13 \mathrm{kPa}$ ). Values for hydrogels with $25 \%$ and $75 \%$ of silk fell in between, being $E_{m}$ of $1.05 \pm 0.15 \mathrm{kPa}$ and $1.14 \pm$ $0.03 \mathrm{kPa}$, respectively. One-way ANOVA revealed a statistical significant effect of silk in the microscale stiffness of the hydrogels $(p=0.019)$, which was also significant when comparing each individual concentration with the pure collagen control, as shown in Figure 3.

\section{Macroscale mechanical properties}

At $20 \%$ strain, control samples showed a stress of $0.70 \pm$ $0.08 \mathrm{kPa}$ and a macroscale elastic modulus $E_{M}$ of $9.93 \pm$ $2.50 \mathrm{kPa}$. The macroscale mechanical properties were 


\section{a}

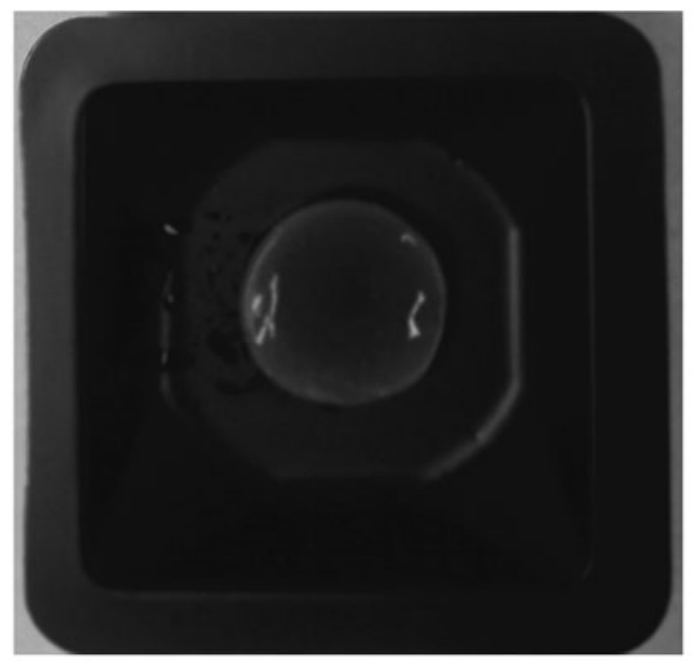

b
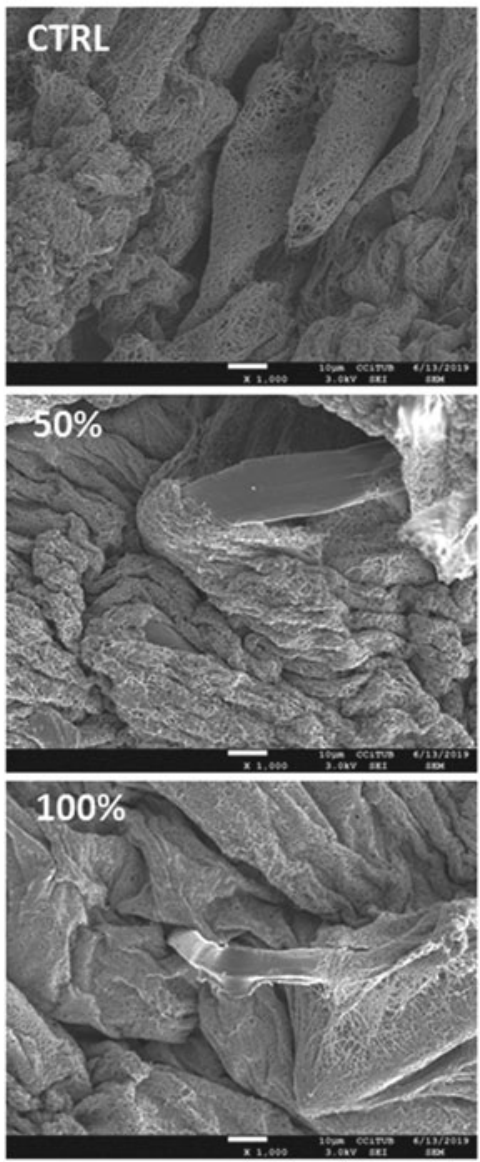
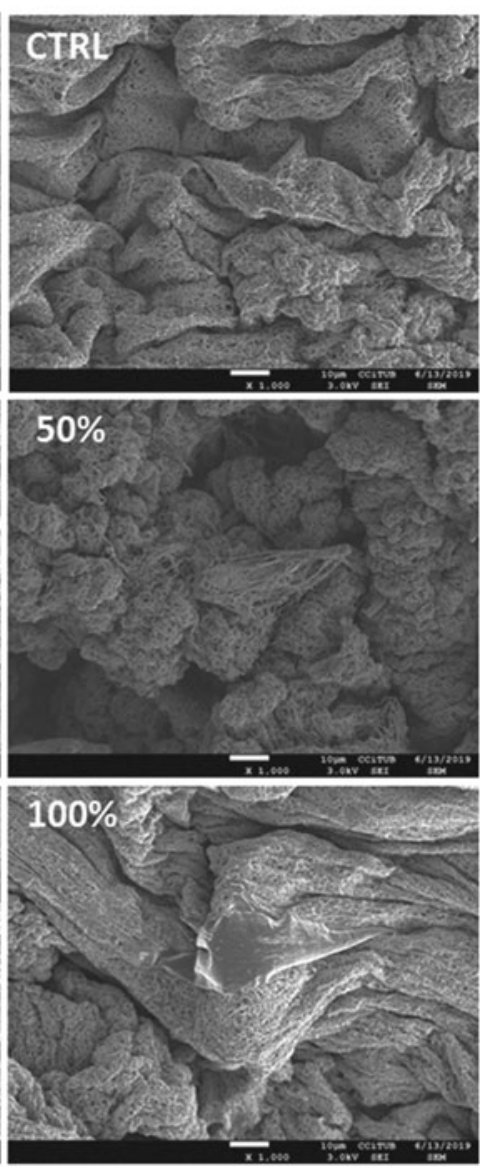

C

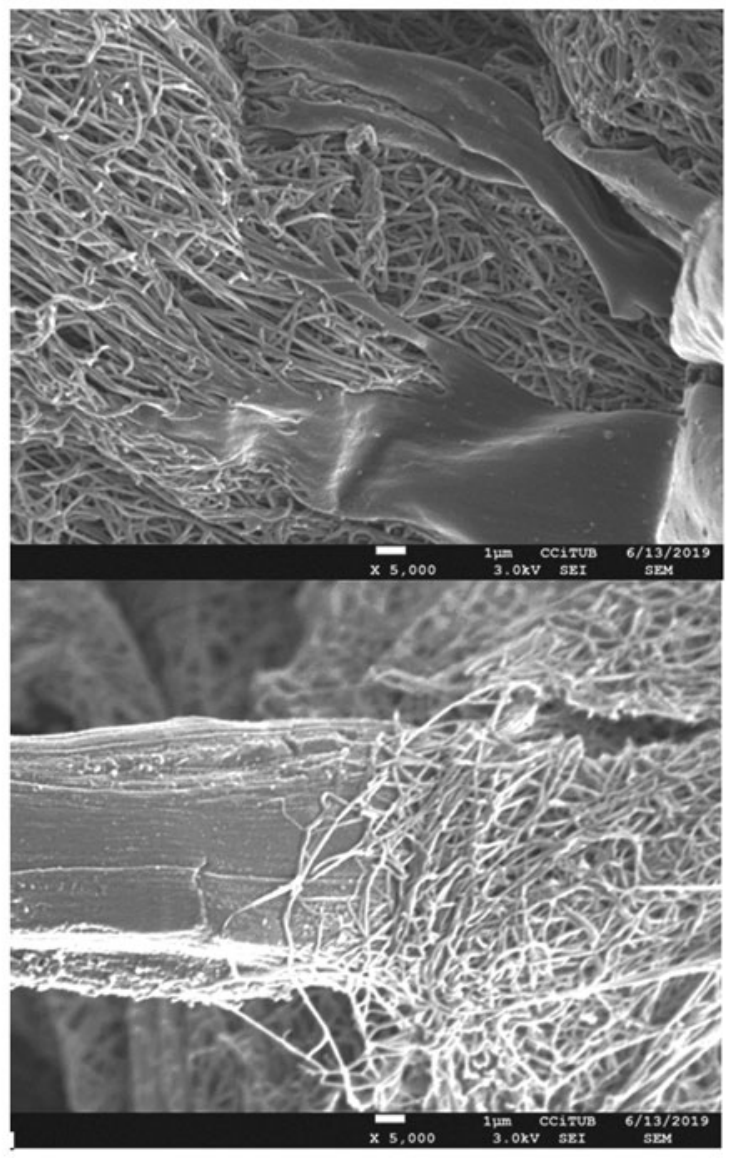

FIG. 1. Ultrastructure images acquired by scanning electron microscopy. (a) Photograph of the $100 \%$ silk-containing (with respect to collagen) hydrogels showing that there is no phase separation in the scaffolds between collagen and silk. (b) Different acellular and cellularized scaffolds. (c) Details of the 100\% images show how the cell was making junctions with the collagen fibers and the silk microfiber is well integrated within the collagen hydrogel.

increased by more than twofold in the silk-reinforced hydrogels, increasing with the silk concentration up to a maximum that was found at the $75 \%$ (stress of $1.77 \pm$ $0.05 \mathrm{kPa}$ and $E_{M}$ of $\left.31.16 \pm 2.96 \mathrm{kPa}\right)$ and decreasing in the $100 \%$ of silk content (stress of $1.11 \pm 0.11 \mathrm{kPa}$ and $E_{M}$ of $18.42 \pm 2.22 \mathrm{kPa}$ ). Values at $25 \%$ and $50 \%$ concentrations fell in between of the control and the $75 \%$ (stress $=1.24 \pm$
$0.05 \mathrm{kPa}$ and $E_{M}=19.34 \pm 3.41 \mathrm{kPa}$ for the $25 \%$ and stress $=$ $1.43 \pm 0.34 \mathrm{kPa}$ and $E_{M}=23.89 \pm 5.82$ for the $50 \%$ ).

One-way ANOVA revealed a statistically significant effect of silk in the macroscale stress $(p<0.001)$ and $E_{M}$ $(p<0.001)$ at $20 \%$ of strain of the hydrogels, and for all the groups, as shown in Figure $4 \mathrm{~b}$. Compression test results agree with the behavior observed for the tensile tests, with 
calculated elastic modulus of $2.6 \mathrm{kPa}(50 \%$ silk content), $2.3 \mathrm{kPa}(100 \%$ silk content), and $1.6 \mathrm{kPa}$ (control).

\section{Rheological characterization}

Viscosity and shear modulus increased with the incorporation of the silk into the hydrogels (Figure 5). Interestingly, at $4^{\circ} \mathrm{C}$ they remain constant for the silk-reinforced hydrogels, while increasing in the pure collagen hydrogels.

\section{Bone marrow derived-mesenchymal stem cells $3 D$ culture within the scaffolds}

A high number of viable BM-MSCs were observed at both $4 \mathrm{~h}$ and 7 days within the collagen scaffolds and no remarkable difference was found for cells cultured in the $50 \%$ silk-incorporated scaffolds and with $100 \%$ of silk content. Cell distribution was homogeneous in the three directions of the scaffolds as shown in Figure 6. Morphology of the cells cultured in the silk-reinforced scaffolds for 7 days was more elongated with respect to the pure collagen control as shown in Figures 6 and 7.

\section{Discussion}

This study presents a novel physical method to solubilize and mix silk from $B$. mori cocoons and type I collagen from rat tails. This straightforward method produces stiffened and stable hydrogel structures that are suitable for 3D bioprinting and culturing of MSCs. Interestingly, incorporation of silk microfibers into the collagen hydrogels results in a twofold increase of their macro- and micromechanical stiffness with no induction of phase separation between the materials nor affecting cell viability in the 3D cultures.

The hydrogels preparation method described here is highly compatible with tissue engineering applications due to the preservation of biocompatibility since no hazardous reagents or high temperatures were used. Cryogenic milling is a physical technique that is widely used in the preparation of extracellular matrix (ECM)-based biomaterials, ${ }^{40}$ and alkaline buffer solubilization of silk is a common procedure. ${ }^{41}$ Moreover, acid extraction of type I collagen is known to better preserve telopeptides in the tropocollagen molecules than pepsin-based solubilization, ${ }^{42}$ and thus it produces collagen fibrils with enhanced capabilities to interact between them and with other proteins, ${ }^{43}$ as confirmed by SEM images.

In keeping with the aim of the study, silk-reinforced collagen scaffolds were produced (maximum silk concentration of $100 \%$ with respect to collagen concentration) instead of collagen-functionalized silk scaffolds. A multiscale mechanical analysis was carried out to assess the relationship between the increase in hydrogel stiffness and silk content, since this is an adequate methodology to obtain a complete picture of the mechanical response of ECM and ECM-mimicking materials. ${ }^{44} \mathrm{BM}$-MSCs, which are nowadays one of the gold standards in regenerative medicine due to their translational potential, ${ }^{45,46}$ were cultured within the scaffolds and a high number of viable cells were observed after 1 week.

Type I collagen is a good scaffold for 3D cell culture, and the incorporation of silk in the preparation of the hydrogels showed an improvement in their properties in the presented multiscale study. Some authors have reported methods for

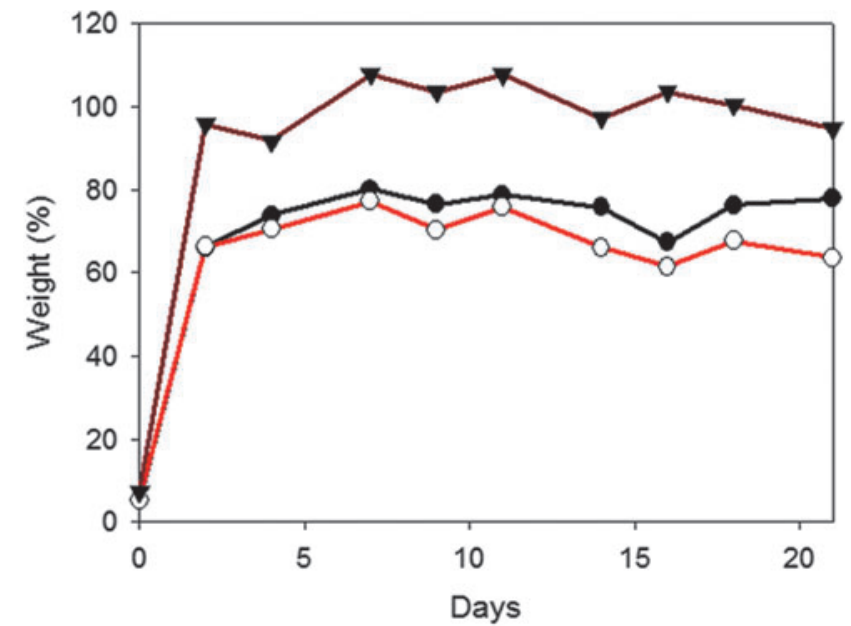

FIG. 2. Swelling and degradation for the control (black), $50 \%$ (red), and 100\% (brown) silk content hydrogels. At time 0 , the hydrogel was freeze dried, and the swelling is expressed in percentage with respect to the original weight before freeze drying. Color images are available online.

mixing type I collagen and purified silk fibroin or degummed silk cocoons (without sericin). ${ }^{22,47}$ However, using silk fibroin alone limits the mixture to have low concentrations in collagen, and phase separation between the materials is often observed. The improvement of blending (by inducing electrostatic interactions between the two polymers) is usually carried out by methods requiring considerable changes in temperature, ${ }^{48}$ complex techniques such electrospinning, ${ }^{49}$ or using additional crosslinkers. ${ }^{50}$ Nevertheless, these procedures may result in poor performance or even in denaturation of type I collagen. In addition, phase separation has been observed when mixing collagen with degummed silk cocoons ${ }^{47}$ when no additional crosslinkers, such as horseradish peroxidase or genipin, were used to create additional bonds in the structure. . $^{5-53}$

The ultrastructural images of the hydrogels developed in this study showed that there is no phase separation between the two biomaterials or denaturation of the collagen fibrils ${ }^{54}$

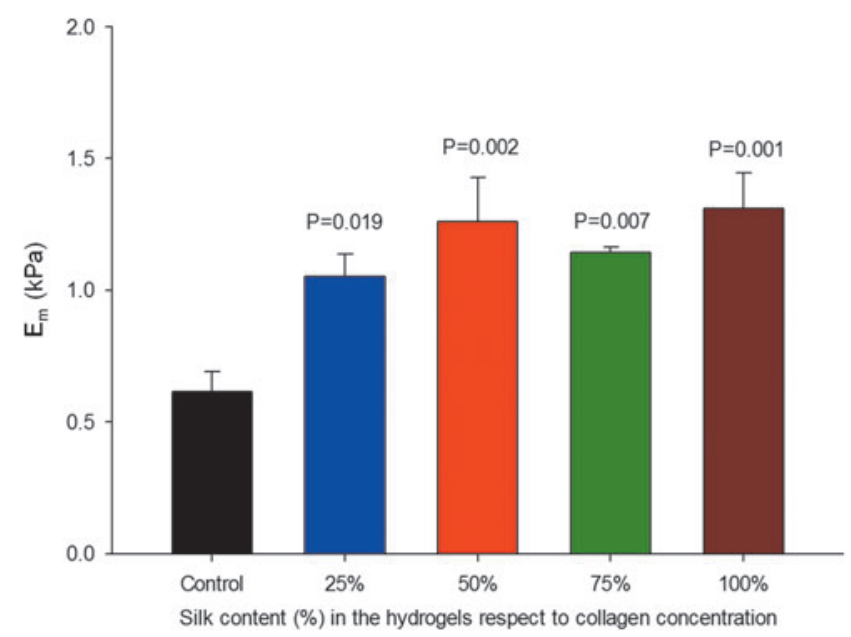

FIG. 3. Local elastic modulus measured with atomic force microscopy $\left(E_{m}\right)$ for the different silk content in the hydrogels. Color images are available online. 
or silk microfibers. As fibroin is mainly hydrophobic ${ }^{55}$ and sericin is highly hydrophilic, ${ }^{56}$ the presence of sericin in the developed hydrogels may help in the formation of the structures due to its water retention capability, ${ }^{57}$ confirmed by the swelling results (Fig. 2). In fact, it has been reported that sericin addition improves the stability of collagen fibrils. ${ }^{58}$ The presence of the hydrophilic serine from sericin ${ }^{56}$ could aid the interaction between the alanine from the fibroin beta sheets ${ }^{59}$ and the proline from the collagen triple helix, because both amino acids are of hydrophobic nature. Therefore, the presence of sericin in the hydrogels seems to help in forming the silk microfiber structures and their interaction with the collagen hydrogel observed in the SEM images (Fig. 1).

Some studies have shown that composite silk scaffolds, developed by mixing fibers and hydrogels, have an impact in the robustness of the constructs, ${ }^{60}$ which is in concor- dance with the results obtained in this study for the silkcollagen composite scaffolds.

Macroscale mechanics of the developed hydrogels was studied by uniaxial tensile testing and bulk compression. By using a bioprinted mold in pluronic to cast the hydrogels before gelation, samples with very similar geometries were obtained, thereby reducing the variability and artifacts in the experiments. Differences in the macromechanics were quantified by the stress and elastic modulus at $20 \%$ strain, which is a strain with physiological relevance in biological application such those involving cardiac ${ }^{61}$ and respiratory ${ }^{62}$ diseases. The values obtained for pure collagen strips were in agreement with previously reported data. ${ }^{63}$ Reinforcing collagen with silk microfibers showed an increase in the values of the stress and elastic modulus up to a maximum found at the $75 \%$ of silk concentration, where the increase was between two- and threefold with respect to the control (Figs. 3 and 4).

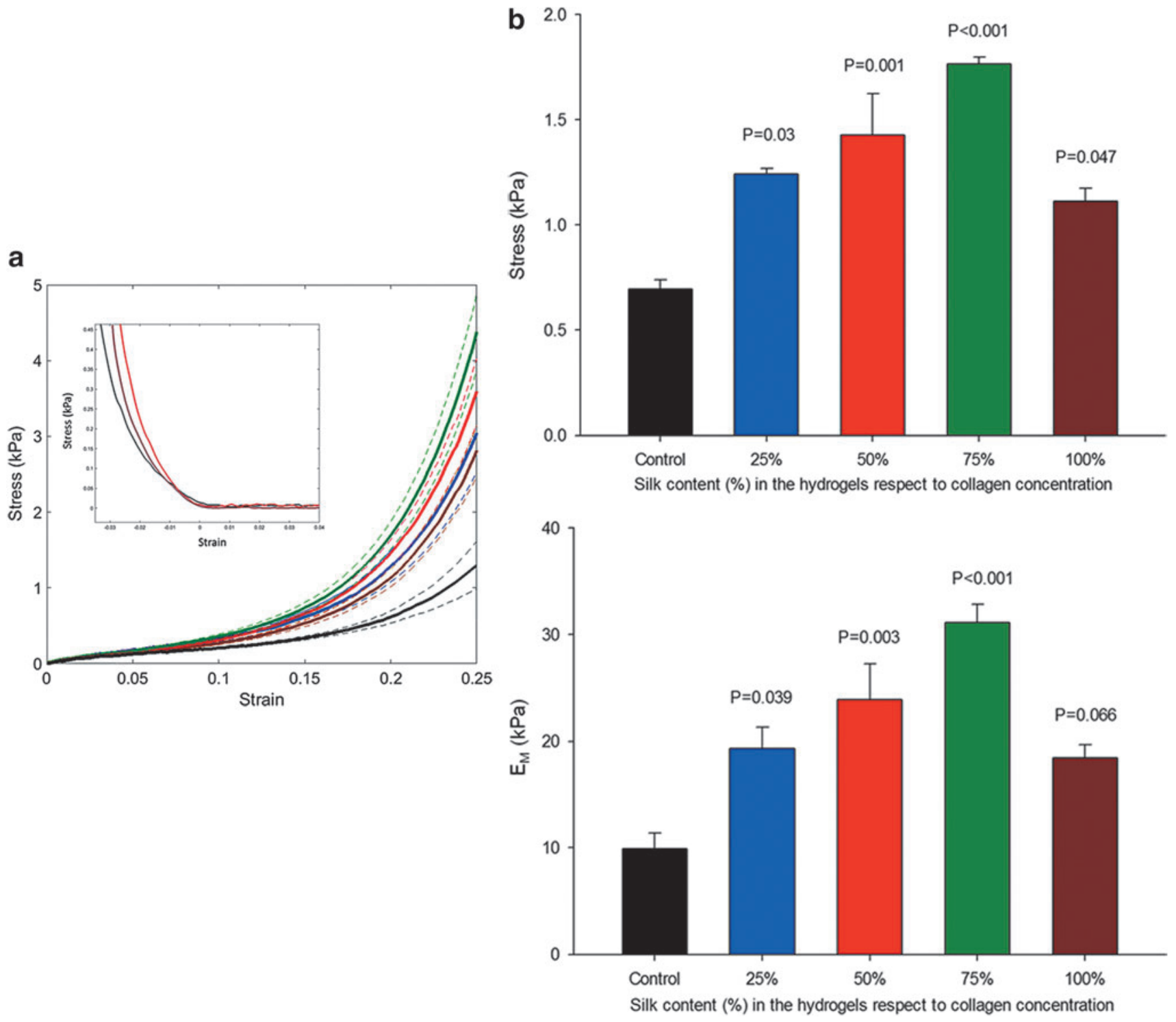

FIG. 4. Macroscale mechanical properties of the hydrogels. (a) Strain-stress curves for the control (black), 25\% (blue), $50 \%$ (red), $75 \%$ (green), and 100\% (brown) silk content. Data are mean (solid lines) \pm standard error (dashed lines). Bulk compression data are shown in the inset for control and silk concentration of $50 \%$ and $100 \%$. (b) Stress and elastic modulus $\left(E_{M}\right)$ at $20 \%$ of strain. Color images are available online. 

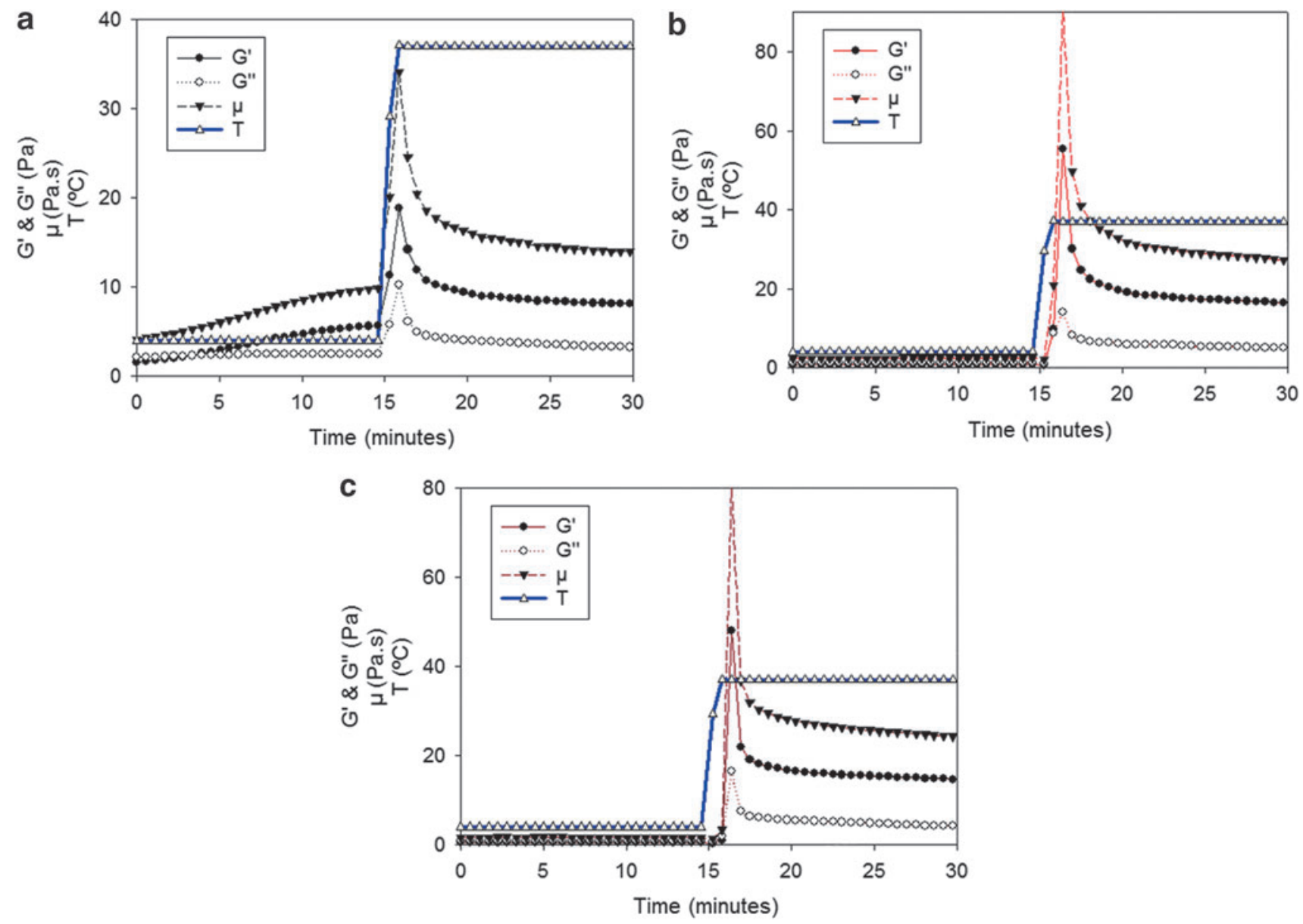

FIG. 5. Rheological data (storage $\left[G^{\prime}\right]$ and loss $\left[G^{\prime \prime}\right]$ moduli, and dynamic viscosity $[\mu]$ ). Temperature $(T)$ was varied from 4 to $37^{\circ} \mathrm{C}$. (a) Collagen control. (b) $50 \%$ of silk content. (c) $100 \%$ of silk content. Color images are available online.

The appearance of a maximum augmentation of elastic modulus for a given silk concentration was already observed in degummed and powdered silk cocoons but with much lower increased stiffness and lower elongation (the samples broke at $\sim 20 \%$ strain, whereas in our experiments, no samples was broken at $30 \%$ strain test). ${ }^{47}$ These results are in keeping with the concept that sericin contributes to building the structure. Microscale mechanics of the hydrogels were assessed by AFM, which is the best-suited technique for mechanical measurements in the length scale at which cells sense their micromechanical niche. ${ }^{64}$ The results we obtained are consistent with those measured by the macroscale measurements, with an augmentation of the microscale elastic modulus of about twofold in between the $50 \%$ and $100 \%$ of silk content (Fig. 1). Therefore, it seems that the observed increase in macroscopic stiffness is related to the microstructure of the hydrogel and to the good integration between fibrils and microfibers due to the presence of sericin.

The rise in stiffness (between two- and threefold) achieved with the new method proposed here could seem a modest improvement, but it is of high importance as the main protein in the scaffolds is still type I collagen. Moreover, the ability to tune the mechanical properties without substantially changing the concentration of collagen is of high importance in modeling healthy and diseased soft tissues, as it is well known that mechanical communication in cells is crucial. ${ }^{65}$ For example, it has been reported that the microscale elastic modulus of lung parenchyma is $\sim 0.7$ $1.5 \mathrm{kPa},{ }^{66}$ but it can be increased twofold with aging. ${ }^{67}$ Moreover, the macroscale elastic modulus at $20 \%$ of strain of healthy mouse myocardium has been reported to increase by twofold when the animals were subjected to severe obstructive sleep apnea. ${ }^{39}$

In the case of cardiac patches, increase in scaffold stiffness is needed so that they can be manipulated, sutured, and glued to the native myocardium, but they should be soft enough to not alter the normal function of the heart. It has been reported that the stress at 0.2 strain of the ventricular myocardium in the longitudinal direction is $\sim 4 \mathrm{kPa}^{68}$ and the elastic modulus at this strain is $\sim 20 \mathrm{kPa}^{69}$ Collagen scaffolds do not exhibit these mechanical properties. By contrast, the silk-reinforced scaffolds have mechanical properties better resembling those of myocardium. Consequently, they could be good candidates for the development of cardiac patches, since they overcome the common problems related to the mechanical instability of naturally derived biomaterials and to the poor biocompatibility of synthetic materials, ${ }^{70}$ which often result in problems such as fibrosis, arrhythmias, and heart failure requiring resurgery as the only viable solution.

In contrast, it is well known that even slight changes in the stiffness of the cell culture microenvironment could result in important changes in stem cell fate. ${ }^{71-73}$ It is worth 
mentioning that the values of stiffness obtained for the pure collagen scaffolds are comparable with the those found in embryos, whereas the values corresponding to the silkreinforced scaffolds are more resembling to adult soft organs such as lung and heart. ${ }^{5,74}$ Therefore, the hydrogels presented in this study could be a good model for in vitro development studies.

Another important point regarding the tunability of scaffold stiffness is the possibility to optimize the cell microenvironment to optimize the paracrine effects of MSCs for further implantation in a recellularized patch for cardiac regeneration. The morphology of the cells cultured in the scaffolds was qualitatively different when silk microfibers were present (Figs. 6 and 7), which could be caused either by the presence of silk or by the stiffening of the hydrogel. Notwithstanding, BM-MSCs cultured in silk-reinforced scaffolds for 7 days showed a more elongated morphology, which has been recently reported as a predictor of their immunosuppressive capacity. ${ }^{75}$ Although out of scope of this study, it is worth noting that silk-reinforced collagen scaffolds could be of interest for preconditioning MSCs. ${ }^{76,77}$

The printability of the developed hydrogels was assessed by rheometry. Rhelogical properties of collagen pregels at $4^{\circ} \mathrm{C}$ changed over time. These changes complicate their use
FIG. 6. Live confocal images of the bioprinted bone marrow-derived mesenchymal stem cells cultured within $1.27 \mathrm{~mm} \times 1.27 \mathrm{~mm} \times$ $200 \mu \mathrm{m}$ scaffolds for $4 \mathrm{~h}$ and 7 days; $Z$ projection in the center and $\mathrm{XZ}$ and $\mathrm{YZ}$ views in the sides. (a) Control collagen. (b) $50 \%$ of silk with respect to collagen protein. (c) $100 \%$ of silk with respect to collagen protein. Color images are available online. a
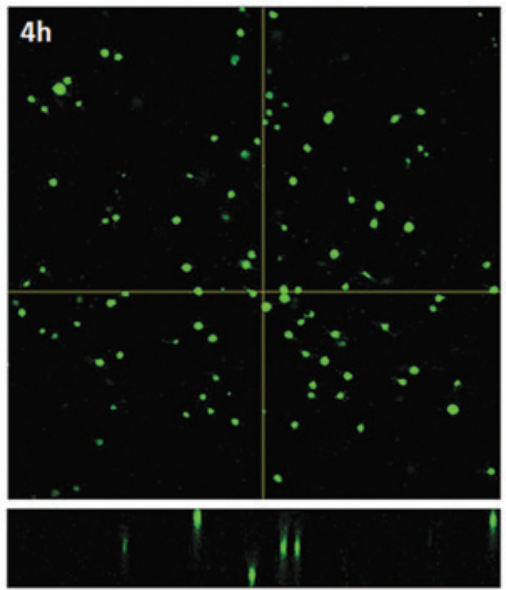

b

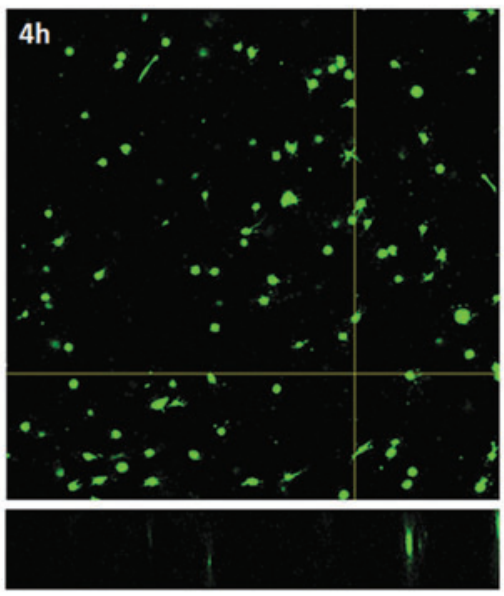

C 4 h

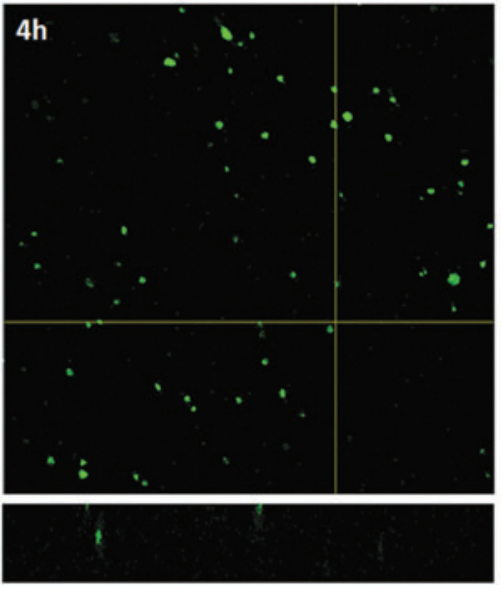

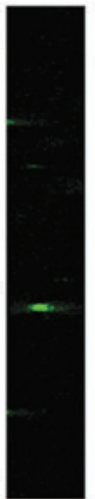
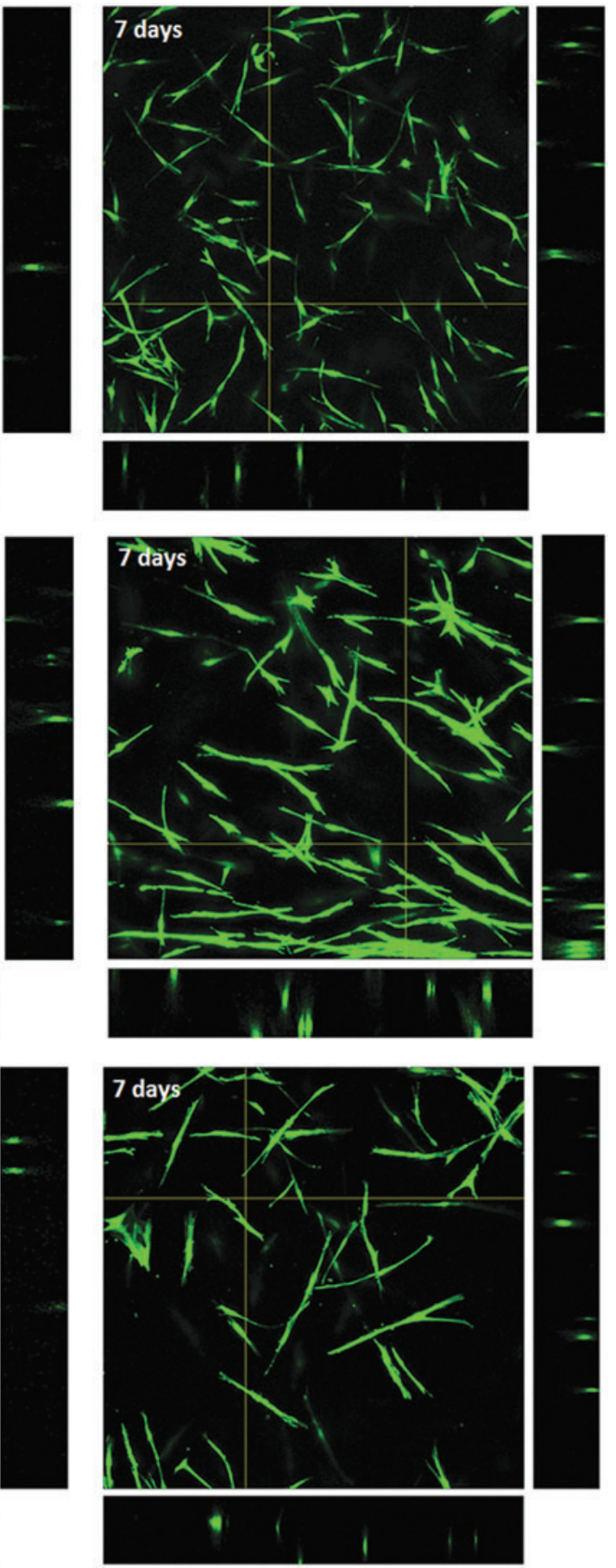

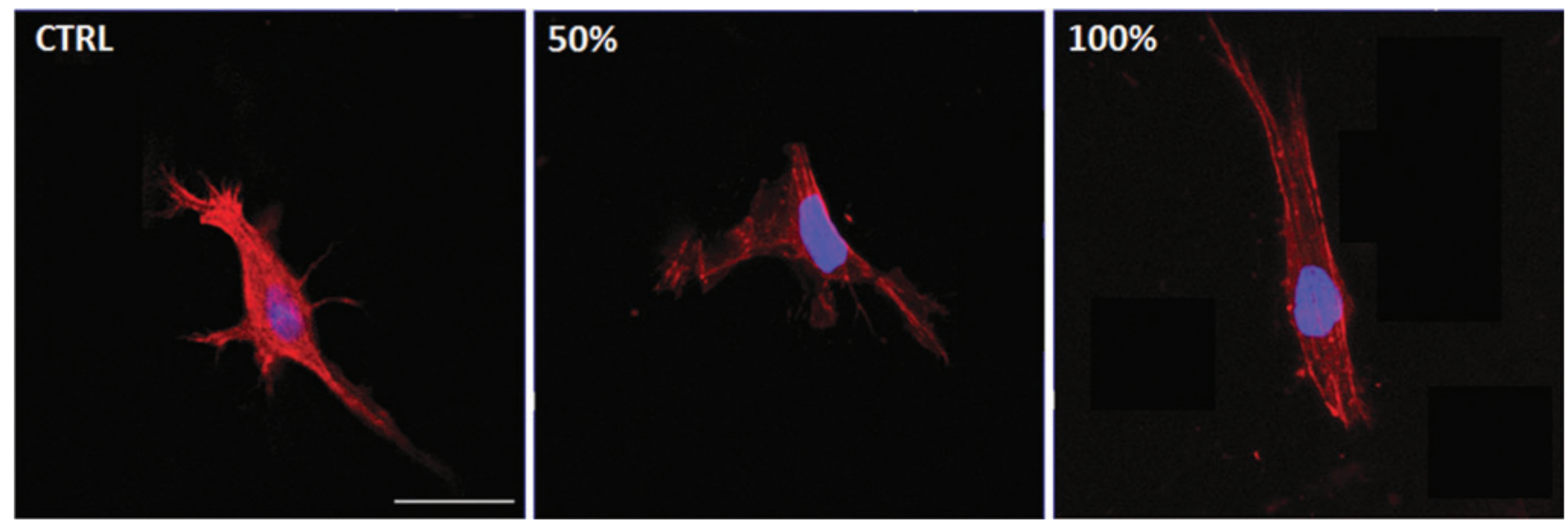

FIG. 7. Images of the cells within the different hydrogels. Scale bar: $100 \mu \mathrm{m}$. Color images are available online.

as a $3 \mathrm{D}$ printing bioink. By contrast, by adding silk to the hydrogels, rheological properties remain stable at low temperature, so the printability of the developed hydrogels is superior to that of collagen hydrogels. This improved printability characteristics will facilitate the development of personalized cardiac patches and the high-throughput fabrication of scaffolds for MSCs preconditioning for further therapeutic applications.

A high number of BM-MSCs were alive within the scaffolds after $3 \mathrm{D}$ bioprinting after 7 days of 3D culture (Fig. 6). It has been reported that the presence of sericin in silk scaffolds favors the viability of cultured cells, ${ }^{78}$ with endothelial cells surviving twofold more when cultured in silk with lower sericin removal. As the live/dead technique is not intended for quantifying the evolution of cell viability along a period of time, the results show that the main objective for the development of cardiac patches and scaffolds for MSCs preconditioning was accomplished.

This study focused on the development of a simple method to develop collagen-silk scaffolds for 3D bioprinting and cell culture. As already mentioned, preservation of sericin in the silk seems to improve the overall performance of the structures. However, further research is required to optimize sericin-fibroin ratio in the scaffolds, as the proportion that is found in the native silkworm cocoons (3:1 to $4: 1$ of fibroinsericin, depending on farming conditions $)^{79}$ has been used in this study. Since protocols to extract fibroin ${ }^{41}$ and sericin, ${ }^{37}$ and to process them to form hydrogels, have been reported, it would be possible to modify the ratio between both proteins in the scaffolds. In contrast, type I collagen ${ }^{80}$ and silk are being used in 3D bioprinting applications, and several techniques to build the scaffolds layer-by-layer have been reported. ${ }^{81,82}$

The developed hydrogels can be effectively bioprinted if the adequate technique is finely tuned (using a pluronic supporting structure in our case) and, when compared with collagen pregels, present the advantage of stable rheological properties when maintained at $4^{\circ} \mathrm{C}$. One of the most promising techniques is FRESH (freeform reversible embedding of suspended hydrogels), where the pregels are printed in liquid phase within a gelatin-supporting structure that is further removed after the gelation of the scaffold, ${ }^{83}$ because the technique can be implemented just by using one printing nozzle.
Potential translation and applicability of the results of this study into the clinics is also promising. For preconditioning of MSCs before cell therapy applications, protocols for the cells to be produced under GMP (good manufacturing practice) have been adapted in the past. ${ }^{84}$ In the case of cardiac patches, the protocol described here will need to be adapted to GMP fabrication, but there are several protocols for producing collagen, ${ }^{85}$ and silk is currently used in the clinical practice for sutures, hence translating the developed technology could be feasible.

\section{Conclusions}

The results presented in this study show that the hydrogels developed by mixing milled raw silkworm cocoons and rat tail type I collagen can be used as bioink to obtain scaffolds for 3D bioprinting in tissue engineering applications such as development of cardiac patches and scaffolds for MSCs preconditioning. Their mechanical properties are improved to better resemble the structure of soft tissues. The method does not require toxic chemicals, additional crosslinkers, or complex physical methods, hence the procedure could be of high interest for further studies in $3 \mathrm{D}$ cell culture and bioprinting. The viability of BM-MSCs when incorporating the silk into the collagen scaffold suggests that the presented biomaterial can be used as a platform for in vitro studies where collagen scaffolds with increased stiffness are required. Also, the improved printability of the biomaterial allows high-throughput fabrication of scaffolds to obtain the high number of cells required for therapy, thereby potentially facilitating translation into clinical practice.

\section{Acknowledgments}

The authors thank Mr. Miguel A. Rodríguez and Ms. Elisabet Urrea (Unit of Biophysics and Bioengineering) for their excellent technical assistance, Dr. Mateu Pla and Dra. Ramona Bravo (Institute for Bioengineering of Catalonia) for their aid with the 3D bioprinter, and Dr. Josep Rebled and Dra. Eva Prats (Scientific Services of the University of Barcelona) for their aid with the SEM.

\section{Disclosure Statement}

No competing financial interests exist. 


\section{Funding Information}

This work was supported in part by the Spanish Ministry of Sciences, Innovation and Universities (DPI2017-83721-P and PGC2018-097323-A-I00) and by the Marie SklodowskaCurie Action, Innovative Training Networks 2018, EU Grant Agreement no. 812772.

\section{References}

1. Mir, T.A., and Nakamura, M. Three-dimensional bioprinting: toward the era of manufacturing human organs as spare parts for healthcare and medicine. Tissue Eng Part B Rev 23, 245, 2017.

2. Zhang, Y.S., and Khademhosseini, A. Advances in engineering hydrogels. Science 356, eaaf3627, 2017.

3. Yannas, I.V., Tzeranis, D.S., Harley, B.A., and So, P.T. Biologically active collagen-based scaffolds: advances in processing and characterization. Philos Trans A Math Phys Eng Sci 368, 2123, 2010.

4. Helary, C., Bataille, I., Abed, A., et al. Concentrated collagen hydrogels as dermal substitutes. Biomaterials 31, 481, 2010.

5. Smith, L., Cho, S., and Discher, D.E. Mechanosensing of matrix by stem cells: from matrix heterogeneity, contractility, and the nucleus in pore-migration to cardiogenesis and muscle stem cells in vivo. Semin Cell Dev Biol 71, 84, 2017.

6. Streeter, B.W., and Davis, M.E. Therapeutic cardiac patches for repairing the myocardium. Adv Exp Med Biol 1144, 1, 2019.

7. Singh, A., and Sen, D. Mesenchymal stem cells in cardiac regeneration: a detailed progress report of the last 6 years (2010-2015). Stem Cell Res Ther 7, 82, 2016.

8. Zhang, J., Zhu, W., Radisic, M., and Vunjak-Novakovic, G. Can we engineer a human cardiac patch for therapy? Circ Res 123, 244, 2018.

9. Bell, D., Prabhu, S., Betts, K., et al. Durability of tissueengineered bovine pericardium $\left(\mathrm{CardioCel}^{\circledR}\right)$ for a minimum of 24 months when used for the repair of congenital heart defects. Interact Cardiovasc Thorac Surg 28, 284, 2018.

10. Tao, Z.W., Wu, S., Cosgriff-Hernandez, E.M., and Jacot, J.G. Evaluation of a polyurethane-reinforced hydrogel patch in a rat right ventricle wall replacement model. Acta Biomater 101, 206, 2019.

11. Darnell, M., O’Neil, A., Mao, A., Gu, L., Rubin, L.L., and Mooney, D.J. Material microenvironmental properties couple to induce distinct transcriptional programs in mammalian stem cells. Proc Natl Acad Sci U S A 115, E8368, 2018.

12. Hirata, M., and Yamaoka, T. Effect of stem cell niche elasticity/ECM protein on the self-beating cardiomyocyte differentiation of induced pluripotent stem (iPS) cells at different stages. Acta Biomater 65, 44, 2018.

13. Nonaka, P.N., Falcones, B., Farre, R., Artigas, A., Almendros, I., and Navajas, D. Biophysically preconditioning mesenchymal stem cells improves treatment of ventilatorinduced lung injury. Arch Bronconeumol 2019 [Epub ahead of print]; DOI: 10.1016/j.arbres.2019.08.014.

14. Murphy, S.V., Skardal, A., and Atala, A. Evaluation of hydrogels for bio-printing applications. J Biomed Mater Res A 101, 272, 2013.

15. Giménez, A., Uriarte, J.J., Vieyra, J., Navajas, D., and Alcaraz, J. Elastic properties of hydrogels and decellular- ized tissue sections used in mechanobiology studies probed by atomic force microscopy. Microsc Res Tech 80, 85, 2017.

16. Holder, A.J., Badiei, N., Hawkins, K., Wright, C., Williams, P.R., and Curtis, D.J. Control of collagen gel mechanical properties through manipulation of gelation conditions near the sol-gel transition. Soft Matter 14, 574, 2018.

17. Sundararaghavan, H.G., Monteiro, G.A., Lapin, N.A., Chabal, Y.J., Miksan, J.R., and Shreiber, D.I. Genipininduced changes in collagen gels: correlation of mechanical properties to fluorescence. J Biomed Mater Res A 87, 308, 2008.

18. Delgado, L.M., Fuller, K., and Zeugolis, D.I. Collagen cross-linking: biophysical, biochemical, and biological response analysis. Tissue Eng Part A 23, 1064, 2017.

19. Liu, C., Lewin Mejia, D., Chiang, B., Luker, K.E., and Luker, G.D. Hybrid collagen alginate hydrogel as a platform for 3D tumor spheroid invasion. Acta Biomater 75, 213, 2018.

20. Fu, S., Ni, P., Wang, B., et al. Injectable and thermosensitive PEG-PCL-PEG copolymer/collagen/n-HA hydrogel composite for guided bone regeneration. Biomaterials 33, 4801, 2012.

21. Buitrago, J.O., Patel, K.D., El-Fiqi, A., et al. Silk fibroin/ collagen protein hybrid cell-encapsulating hydrogels with tunable gelation and improved physical and biological properties. Acta Biomater 69, 218, 2018.

22. Long, K., Liu, Y., Li, W., et al. Improving the mechanical properties of collagen-based membranes using silk fibroin for corneal tissue engineering. J Biomed Mater Res A 103, 1159, 2015.

23. Zhang, X., Zhai, C., Fei, H., et al. Composite silkextracellular matrix scaffolds for enhanced chondrogenesis of mesenchymal stem cells. Tissue Eng Part C Methods 24, 645, 2018

24. Steiner, D., Lang, G., Fischer, L., et al. Intrinsic vascularization of recombinant eADF4(C16) spider silk matrices in the arteriovenous loop model. Tissue Eng Part A 25, 1504, 2019.

25. Affas, S., Schäfer, F.M., Algarrahi, K., et al. Augmentation cystoplasty of diseased porcine bladders with bi-layer silk fibroin grafts. Tissue Eng Part A 25, 855, 2019.

26. Frauchiger, D.A., Tekari, A., Wöltje, M., Fortunato, G., Benneker, L.M., and Gantenbein, B. A review of the application of reinforced hydrogels and silk as biomaterials for intervertebral disc repair. Eur Cell Mater 34, 271, 2017.

27. Altman, G.H., Diaz, F., Jakuba, C., et al. Silk-based biomaterials. Biomaterials 24, 401, 2003.

28. Thurber, A.E., Omenetto, F.G., and Kaplan, D.L. In vivo bioresponses to silk proteins. Biomaterials 71, 145, 2015.

29. Font Tellado, S., Bonani, W., Balmayor, E.R., et al. Fabrication and characterization of biphasic silk fibroin scaffolds for tendon/ligament-to-bone tissue engineering. Tissue Eng Part A 23, 859, 2017.

30. Cai, H., Wu, B., Li, Y., et al. Local delivery of silkcellulose incorporated with SDF-1 $\alpha$ functionally improves the uterus repair. Tissue Eng Part A 25, 1514, 2019.

31. Yang, Y., Chen, X., Ding, F., Zhang, P., Liu, J., and Gu, $\mathrm{X}$. Biocompatibility evaluation of silk fibroin with peripheral nerve tissues and cells in vitro. Biomaterials 28, 1643, 2007.

32. Liu, B., Song, Y.W., Jin, L., et al. Silk structure and degradation. Colloids Surf B Biointerfaces 131, 122, 2015. 
33. Lamboni, L., Gauthier, M., Yang, G., and Wang, Q. Silk sericin: a versatile material for tissue engineering and drug delivery. Biotechnol Adv 33, 1855, 2015.

34. Sapru, S., Das, S., Mandal, M., Ghosh, A.K., and Kundu, S.C. Nonmulberry silk protein sericin blend hydrogels for skin tissue regeneration-in vitro and in vivo. Int $\mathrm{J}$ Biol Macromol 137, 545, 2019.

35. Rajan, N., Habermehl, J., Coté, M.F., Doillon, C.J., and Mantovani, D. Preparation of ready-to-use, storable and reconstituted type I collagen from rat tail tendon for tissue engineering applications. Nat Protoc 1, 2753, 2006.

36. Wolf, M.T., Daly, K.A., Brennan-Pierce, E.P., et al. A hydrogel derived from decellularized dermal extracellular matrix. Biomaterials 33, 7028, 2012.

37. Wang, Z., Zhang, Y., Zhang, J., et al. Exploring natural silk protein sericin for regenerative medicine: an injectable, photoluminescent, cell-adhesive 3D hydrogel. Sci Rep 4, 7064, 2014.

38. Alcaraz, J., Otero, J., Jorba, I., and Navajas, D. Bidirectional mechanobiology between cells and their local extracellular matrix probed by atomic force microscopy. Semin Cell Dev Biol 73, 71, 2018.

39. Farré N, Otero, J., Falcones, B., et al. Intermittent hypoxia mimicking sleep apnea increases passive stiffness of myocardial extracellular matrix. A multiscale study. Front Physiol 9, 1143, 2018.

40. Freytes, D.O., O’Neill, J.D., Duan-Arnold, Y., Wrona, E.A., and Vunjak-Novakovic, G. Natural cardiac extracellular matrix hydrogels for cultivation of human stem cell-derived cardiomyocytes. Methods Mol Biol 1181, 69, 2014.

41. Rockwood, D.N., Preda, R.C., Yücel, T., Wang, X., Lovett, M.L., and Kaplan, D.L. Materials fabrication from Bombyx mori silk fibroin. Nat Protoc 6, 1612, 2011.

42. Meyer, M. Processing of collagen based biomaterials and the resulting materials properties. Biomed Eng Online 18, 24, 2019.

43. Shayegan, M., Altindal, T., Kiefl, E., and Forde, N.R. Intact telopeptides enhance interactions between collagens. Biophys J 111, 2404, 2016.

44. Jorba, I., Beltrán, G., Falcones, B., et al. Nonlinear elasticity of the lung extracellular microenvironment is regulated by macroscale tissue strain. Acta Biomater 92, 265, 2019.

45. Mendicino, M., Bailey, A.M., Wonnacott, K., Puri, R.K., and Bauer, S.R. MSC-based product characterization for clinical trials: an FDA perspective. Cell Stem Cell 14, 141, 2014.

46. Samsonraj, R.M., Raghunath, M., Nurcombe, V., Hui, J.H., van Wijnen, A.J., and Cool, S.M. Concise review: multifaceted characterization of human mesenchymal stem cells for use in regenerative medicine. Stem Cells Transl Med 6, 2173, 2017.

47. de Moraes, M.A., Paternotte, E., Mantovani, D., and Beppu, M.M. Mechanical and biological performances of new scaffolds made of collagen hydrogels and fibroin microfibers for vascular tissue engineering. Macromol Biosci 12, 1253, 2012.

48. Lu, Q., Feng, Q., Hu, K., and Cui, F. Preparation of threedimensional fibroin/collagen scaffolds in various $\mathrm{pH}$ conditions. J Mater Sci Mater Med 19, 629, 2008.

49. Zhou, J., Cao, C., Ma, X., and Lin, J. Electrospinning of silk fibroin and collagen for vascular tissue engineering. Int J Biol Macromol 47, 514, 2010.
50. Lv, Q., Hu, K., Feng, Q., and Cui, F. Fibroin/collagen hybrid hydrogels with crosslinking method: preparation, properties, and cytocompatibility. J Biomed Mater Res A 84, 198, 2008.

51. McGill, M., Coburn, J.M., Partlow, B.P., Mu, X., and Kaplan, D.L. Molecular and macro-scale analysis of enzymecrosslinked silk hydrogels for rational biomaterial design. Acta Biomater 63, 76, 2017.

52. Chirila, T.V., Suzuki, S., and Papolla, C. A comparative investigation of Bombyx mori silk fibroin hydrogels generated by chemical and enzymatic cross-linking. Biotechnol Appl Biochem 64, 771, 2017.

53. Liu, C., Jin, Z., Ge, X., Zhang, Y., and Xu, H. Decellularized annulus fibrosus matrix/chitosan hydrogels for annulus fibrous tissue engineering. Tissue Eng Part A 25, 1605, 2019.

54. Antoine, E.E., Vlachos, P.P., and Rylander, M.N. Review of collagen I hydrogels for bioengineered tissue microenvironments: characterization of mechanics, structure, and transport. Tissue Eng Part B Rev 20, 683, 2014.

55. Vepari, C., and Kaplan, D.L. Silk as a biomaterial. Prog Polym Sci 32, 991, 2007.

56. Kunz, R.I., Brancalhão, R.M., Ribeiro, L.F., and Natali, M.R. Silkworm sericin: properties and biomedical applications. Biomed Res Int 2016, 8175701, 2016.

57. Ghaeli, I., de Moraes, M.A., Beppu, M.M., et al. Phase behaviour and miscibility studies of collagen/silk fibroin macromolecular system in dilute solutions and solid state. Molecules 22, E1368, 2017.

58. Duan, L., Yuan, J., Yang, X., Cheng, X., and Li, J. Interaction study of collagen and sericin in blending solution. Int J Biol Macromol 93(Pt A), 468, 2016.

59. Hayashi, C.Y., Shipley, N.H., and Lewis, R.V. Hypotheses that correlate the sequence, structure, and mechanical properties of spider silk proteins. Int J Biol Macromol 24, 271, 1999.

60. Yodmuang, S., McNamara, S.L., Nover, A.B., et al. Silk microfiber-reinforced silk hydrogel composites for functional cartilage tissue repair. Acta Biomater 11, 27, 2015.

61. Park, J.H., Lee, J.H., Lee, S.Y., et al. Normal 2dimensional strain values of the left ventricle: a substudy of the normal echocardiographic measurements in Korean population study. J Cardiovasc Ultrasound 24, 285, 2016.

62. Peñuelas, O., Melo, E., Sánchez, C., et al. Antioxidant effect of human adult adipose-derived stromal stem cells in alveolar epithelial cells undergoing stretch. Respir Physiol Neurobiol 188, 1, 2013.

63. Valero, C., Amaveda, H., Mora, M., and García-Aznar, J.M. Combined experimental and computational characterization of crosslinked collagen-based hydrogels. PLoS One 13, e0195820, 2018.

64. Andreu, I., Luque, T., Sancho, A., et al. Heterogeneous micromechanical properties of the extracellular matrix in healthy and infarcted hearts. Acta Biomater 10, 3235, 2014.

65. Sapir, L., and Tzlil, S. Talking over the extracellular matrix: how do cells communicate mechanically? Semin Cell Dev Biol 71, 99, 2017.

66. Liu, F., and Tschumperlin, D.J. Micro-mechanical characterization of lung tissue using atomic force microscopy. J Vis Exp 2911, 2011.

67. Sicard, D., Haak, A.J., Choi, K.M., Craig, A.R., Fredenburgh, L.E., and Tschumperlin, D.J. Aging and anatomical variations in lung tissue stiffness. Am J Physiol Lung Cell Mol Physiol 314, L946, 2018. 
68. Engelmayr, G.C., Cheng, M., Bettinger, C.J., Borenstein, J.T., Langer, R., and Freed, L.E. Accordion-like honeycombs for tissue engineering of cardiac anisotropy. Nat Mater 7, 1003, 2008.

69. Perea-Gil, I., Gálvez-Montón, C., Prat-Vidal, C., et al. Head-to-head comparison of two engineered cardiac grafts for myocardial repair: from scaffold characterization to preclinical testing. Sci Rep 8, 6708, 2018.

70. Pok, S., and Jacot, J.G. Biomaterials advances in patches for congenital heart defect repair. J Cardiovasc Transl Res 4, 646, 2011.

71. Zakrzewski, W., Dobrzyński, M., Szymonowicz, M., and Rybak, Z. Stem cells: past, present, and future. Stem Cell Res Ther 10, 68, 2019.

72. Lane, S.W., Williams, D.A., and Watt, F.M. Modulating the stem cell niche for tissue regeneration. Nat Biotechnol 32, 795, 2014.

73. Žigon-Branc, S., Markovic, M., Van Hoorick, J., et al. Impact of hydrogel stiffness on differentiation of human adipose-derived stem cell microspheroids. Tissue Eng Part A 25, 1369, 2019.

74. Discher, D.E., Mooney, D.J., and Zandstra, P.W. Growth factors, matrices, and forces combine and control stem cells. Science 324, 1673, 2009.

75. Klinker, M.W., Marklein, R.A., Lo Surdo, J.L., Wei, C.H., and Bauer, S.R. Morphological features of IFN- $\gamma$ stimulated mesenchymal stromal cells predict overall immunosuppressive capacity. Proc Natl Acad Sci U S A 114, E2598, 2017.

76. $\mathrm{Hu}, \mathrm{C}$. , and $\mathrm{Li}, \mathrm{L}$. Preconditioning influences mesenchymal stem cell properties in vitro and in vivo. J Cell Mol Med 22, 1428, 2018.

77. Ezquer, F.E., Ezquer, M.E., Vicencio, J.M., and Calligaris, S.D. Two complementary strategies to improve cell engraftment in mesenchymal stem cell-based therapy: increasing transplanted cell resistance and increasing tissue receptivity. Cell Adh Migr 11, 110, 2017.

78. Wray, L.S., Hu, X., Gallego, J., et al. Effect of processing on silk-based biomaterials: reproducibility and biocompatibility. J Biomed Mater Res B Appl Biomater 99, 89, 2011.
79. Zhang, Y., Zhao, D., Meng, Z., et al. Wild silkworm cocoon contains more metabolites than domestic silkworm cocoon to improve its protection. J Insect Sci 17, 2017.

80. Osidak, E.O., Karalkin, P.A., Osidak, M.S., et al. Viscoll collagen solution as a novel bioink for direct 3D bioprinting. J Mater Sci Mater Med 30, 31, 2019.

81. Rodriguez, M.J., Dixon, T.A., Cohen, E., Huang, W., Omenetto, F.G., and Kaplan, D.L. 3D freeform printing of silk fibroin. Acta Biomater 71, 379, 2018.

82. Chawla, S., Midha, S., Sharma, A., and Ghosh, S. Silkbased bioinks for 3D bioprinting. Adv Healthc Mater 7, e1701204, 2018.

83. Hinton, T.J., Jallerat, Q., Palchesko, R.N., et al. Threedimensional printing of complex biological structures by freeform reversible embedding of suspended hydrogels. Sci Adv 1, e1500758, 2015.

84. Viganò, M., Budelli, S., Lavazza, C., et al. Tips and tricks for validation of quality control analytical methods in good manufacturing practice mesenchymal stromal cell production. Stem Cells Int 2018, 3038565, 2018.

85. Hartmann-Fritsch, F., Marino, D., and Reichmann, E. About ATMPs, SOPs and GMP: the Hurdles to produce novel skin grafts for clinical use. Transfus Med Hemother 43, 344, 2016.

Address correspondence to: Jorge Otero, PhD

Unit of Biophysics and Bioengineering School of Medicine and Health Sciences Universitat de Barcelona Casanova 143 Barcelona 08036 Spain

E-mail: jorge.otero@ub.edu

Received: July 31, 2019

Accepted: February 11, 2020

Online Publication Date: March 18, 2020 